Prof. Dr. Cordula Stumpf/Dr. Friedemann Kainer (Hrsg.)

Gemeinschaftsrecht als

Gestaltungsaufgabe

Entwicklungen, Bedingungen, Perspektiven

Geburtstagskolloquium für

Prof. Dr. Dr. h.c. Peter-Christian Müller-Graff Heidelberg, 15. Oktober 2005 


\title{
Strukturen der europäischen Finanz- und Haushaltsverfassung
}

\author{
Priv:-Doz. Dr. Matthias Rossi, Berlin
}

\section{Eimleirung}

Der Konstitutionalisierungsprozess der Europäischen Union ist mit dem Ausgang der Referenden in Frankreich und in den Niederlanden ins Stocken geraten. Dies mag begrüßt oder bedauert werden. darf aber in keinem Fall den Blick dafür verstellen. dass die derzeitigen Verträge jedenfalls in ihren organisationsrechtlichen Bestimmungen einer Novellierung und Reformierung beduirfen, um sowohl die Handlungsfähigkeit als auch die Akzeptanz des transnationalen Gemeinwesens' mit seinen 25, 27 oder 28 Mitgliedstaaten dauerhaft zu gewährleisten. Das gilt insbesondere auch für die finanz- und haushaltsrechtlichen Bestimmungen. die die von den Verträgen vorgenommene Kompetenzverteilung sichern und stützen. ${ }^{2}$ Dass die Finanzordnung das Kernproblem im Verhältnis zwischen der Europäischen Union und ihren Mitgliedstaaten darstellt, verdeutlicht die politische Diskussion um die mittelfristige Finanzplanung für die Jahre 2007 - 2013, die erst mit großer Verspätung und nur durch einen die eigentlichen Probleme ausklammernden Kompromiss im Dezember 2005 vorläufig beendet werden konnte.

Vor diesem Hintergrund soll nachfolgend die vom Vertrag über eine Verfassung für Europa ${ }^{3}$ konstituierte Finanz- und Haushaltsverfassung der Europäischen Union skizziert werden. ${ }^{4}$ Denn dieser Verfassungsvertrag führı nicht nur zu zahlreichen Veränderungen im institutionellen Gefüge der Union und zu einer Neuordnung und verteilung der materiellen Kompetenzen zwischen der Union und den Mitgliedstaaten. sondern modifiziert auch die finanz- und insbesondere die haushaltsrechtlichen Bestimmungen des EG-Vertrags zum Teil erheblich; modifiziert sie in einer Weise, die für die notwendige weitere Konstitutionalisierung ${ }^{5}$ - mag sie durch eine neu ausformulierle und als solche bezeichnete Europäische Verfassung. mag sie durch eine weitreichende Novellierung der geltenden Verträge erfolgen - als Vorbild. jedenfalls aber als Beispiel dienen kann.

1 Zu dieser Charakierisierung der Europäischen Union Mïller-Graff. Europiaische Verfassung und Grundrechtscharta: Die Europäische Union als Iransnationales Gemeinwesen, integration 20100, S. 34 ff.

2 Zu dieser Funktion der Finanzverfassung im Bundesstaat vgl. Wendt. Finanzhoheil und Finanzausgleich. in: Josef Isensee/Paul Kirchhof (Hrsg.). Handbuch des Staatsrechts. Band IV. Heidelberg 1990. S. $1021 \mathrm{ff}$.

3 In Folgenden Verlrag über eine Verfassung für Europa - VVE.

4 Zur detaillienen Kommentierung sämtlicher Finanzvorschriften des VVE vgl. Rossi, in: Vedder/Heintschel von Heinegg (Hrsg.). Europäischer Verfassungsvertrag, im Erscheinen.

5 Vgl. Müller-Graff. Europäische Verfassungsordnung - Notwendigkeit. Gestalt und Fontentwicklung, EWS 2002, S. 206, $209 \mathrm{ff}$. 


\section{Stellung der Haushaltsverfassung im VVE}

Die wesentlichen Bestimmungen der Haushaltsverfassung des VVE finden sich sowohl in dem Definitionen und Ziele enthaltenden Ersten Teil als auch in dem die Politikbereiche und die Arbeitsweise der Union behandelnden Dritten Teil. ${ }^{6}$ Im Ersten Teil normiert der mit „Die Finanzen der Union" überschriebene Titel VII in den Art. 1-53 VVE bis Art. I-56 VVE die grundlegenden Bestimmungen über die Haushalts- und Finanzgrundsätze, die Eigenmittel der Union, den mehrjährigen Finanzrahmen und den Haushaltsplan der Union. Diese Bestimmungen werden im Dritten Teil vom Kapitel II „Finanzvorschriften“ aufgegriffen und konkretisiert. Neben diesen zentralen Vorschriften der Haushaltsverfássung enthält der VVE weitere Bestimmungen, die jedenfalls im materiellen Sinne zur Haushaltsverfassung gezählı werden können. Hervorgehoben werden sollen hier nur die Vorschriften über den Europiischen Rechnungshof in Art. I-31 VVE sowie in Art. III-384 VVE und Art. III-385 VVE.

Die unterschiedliche systematische Stellung der einzelnen Vorschriften wirkt sich vor allem auf ihre Änderbarkeit aus: Während die im Ersten Teil befindlichen Bestimmungen nur im Wege des ordentlichen Änderungsverfahrens nach Art. IV-443 VVE und das heißt nach Ratifizierung in den Mitgliedstaaten geändert werden können. sind die Vorschriften des Dritten Teils auch Änderungen im vereinfachten Verfahren nach Art. IV-444 VVE zugänglich.

\section{Veränderungen im Überblick}

Die zahlreichen Modifizierungen der Haushaltsverfassung des VVE gegenüber den entsprechenden Bestimmungen des EG-Vertrags beruhen vor allem auf zwei grundlegenden Veränderungen:

Zum einen knüpfen die Haushaltsbefugnisse des Rates und des Parlaments nicht mehr an die Unterscheidung zwischen obligatorischen und nicht-obligatorischen Ausgaben an, sondern erstrecken sich gleichermaßen auf alle Ausgaben. Aus diesem Grunde konnten sowohl das reguläre Haushaltsverfahren (Art. III-404 VVE) als auch das Nothaushaltsrecht (Art. III-405 VVE) deutlich vereinfacht werden. Bedeutungslos ist die Unterscheidung zwischen den beiden Ausgabekategorien allerdings nicht geworden, weil Art. III-413 VVE die am Haushaltsverfahren beteiligten Organe verfassungsrechtlich verpflichtet, (vorrangig) die Finanzierbarkeit der obligatorischen Ausgaben sicherzustellen.

Zum anderen sind die Interinstitutionellen Vereinbarungen zwischen der Kommission, dem Rat und dem Parlament, die seit 1988 die rechtzeitige Verabschiedung der Haushaltspläne sowie vor allem die Haushaltsdisziplin gewährleisten, konstitu-

6 Zur Genese dieser Zweiteilung der Finanzbestimmungen vgl. Magiera, Die Finanzordnung im Verfassungsvertrag der Europiiischen Union, in: Jörg Bröhmer u.a. (Hrsg.), Internationale Gemeinschaft und Menschenrechte, Festschrift für Georg Ress zum 70. Geburtstag, Köln 2005, S. $623 \mathrm{ff}$. 
tionalisiert worden. Die in ihnen geregelte finanzielle Vorausschau bestimmı nun als mehrjähriger Finanzrahmen verbindlich die jährlichen Haushaltspläne (Art. I-55, III402 VVE); und der ebenfalls von ihnen vorgesehene Trilog, die Zusammenkunft der Präsidenten der drei am Haushaltsverfahren beteiligten Organe, wird im VVE nun institutionalisjert (Aut. III-414 VVE).

Weitere Veränderungen betreffen die hervorgehobene Bedeutung der Haushaltsgrundsäize (Art. I-53 VVE), die Rechtsform des Haushaltsplans (Arl. 1-56 VVE) sowie in geringerem Maße die Ausfïhrung des Haushaltsplans (Art. III-407 VVE) und das Entlastungsverfahren (Art. III-408, Art. III-409 VVE). Für die Einnahmen des Haushalts bringt der VVE dagegen keine grundsätzlichen Veränderungen: Nach wie vor beschließt der Rat einstimmig iber die Bestimmungen des Eigenmittelsystems, die erst nach Ratifizierung in den Mitgliedstaaten in Kraft treten (Art. I-54 VVE).

Die haushaltsrechtlichen Vorschriften des VVE sind durch eine Reihe weiterer Vorschriften zu ergänzen, die in der Form Europäischer Gesetze auf der Grund lage des VVE zu erlassen sind. Hervorzuheben sind die Eigenmittelgesetze (Art. I-54 VVE), die Finanzrahmengesetze (Art. I-55 VVE) und die Haushaltsordnung (Ar. III-412 VVE). Wegen ihrer determinierenden Bedeutung für die jăhrlich festzulegenden Haushaltspläne sind diese Gesetze (zumindest in Teilen) als materielles Verfassungsrecht zu qualifizieren.

\section{Einnallmen}

In Bezug auf die Einnahmen normient Att. 1-55 Abs. 2 VVE lediglich, dass sich die Europäische Union vollständig aus Ejgenmitteln finanziert. Diese Aussage zielt vor allem auf den Ausschluss einer Finanzierung aus Mitgliedsbeiträgen. Eigenmittel sind mithin solche Mittel, die durch das Recht der EU bestimmt und insoweit der Autonomie der Mitgliedstaaten entzogen sind. ${ }^{\text {. }}$

\section{Festlegung des Systems der Eigenmilte]}

Über die Arl def Eigerunittel enthialt die Verfassung dagegen keine, über ihre Höhe nur eine mittelbare Aussage. Beide Faktoren sind gemáB Art. 1-54 Abs. 3 VVE dem

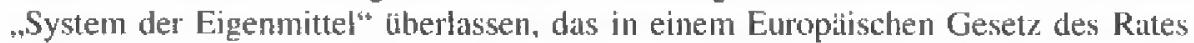
festzulegen ist. Damit wird im Unterschied zu Art. 269 EG nun die Rechtsform bestimmt, in der über das System der Eigenmittel entschieden wird. An dèn zweistufigen Verfahren zum Erlass dieses Ejgenmiltelgesetzes hat sich aber nichts getirdert: Zunäichst entscheidet der Rat einstimmig nach Anhörung des Parlanents iiber das Eigenmittelgesetz, das anschließend von den Mitgliedstaaten nach ihren jeweiligen verfassungsrechilichen Vorschrifien ratifiziert werden muss. Wegen dieses zweistufigen Verfahrens ist das Eigenmittelgesetz nicht ausschließBlich dem Recht

7 Waldhoff, in: Cilliess/Ruffert (Hrsg.), EUV/EGV. 3. Aull. 2007. Art. 269 Rn. 4 m.w. N.; vgl. auch Firgnom, in: Dauses (Hrsg.). Handbuch des EU-Wirtschaftsrechls, A Itl, Rn. I04 tf. 
der Union zuzurechnen; es handelt sich nach wie vor um einen Rechtsakt sui generis. ${ }^{8}$ Immerhin sieht der neu eingelügte und heftig umstrittene ${ }^{y}$ Art. I-54 Abs. 4 VVE nun eine Öffnungsklausel vor, nach der bestimmte Durchführungsmaßnahmen zu dem Eigenmittelsystem vom Rat mit qualifizierter Mehrheit nach Zustimmung des Parlaments und ohne Ratifizierung in den Mitgliedstaaten erlassen werden können. Allerdings muss diese Klausel erst durch das - von den Mitgliedstaaten zu ratifizierende - Eigenmittelgesetz aktivient werden. Und auch nach ihrer Aktivierung wird sich ihre Bedeutung in Grenzen halten. Denn um zu verhindern, dass das Einstimmigkeits- und Ratifizierungserfordernis nach Abs. 3 unterlaufen wird, ist der Begriff der „Durchführungsmaßnahmen“ eng auszulegen und erfasst jedenfalls keine Maßnahmen, mit denen die Art oder die Höhe der Eigenmittel verändert werden sollen.

\section{Arten der Eigenmittel}

Mit Art. 2 Abs. 1 des Eigenmiltelbeschlusses 2000 lassen sich derzeit vier Arten von Eigenmitteln unterscheiden: Agrarabschöpfungen und Zölle als traditionelle Eigenmittel sowie die später geschaffenen Eigenmittel der Mehrwertsteuereinnahmen und Bruttosozialproduktmittel.

Agrarabschöpfungen wurden im Warenverkehr mit Drittstaaten eingeführt. um die Preisunterschiede zwischen den Binnenmarkt- und den Weltmarktpreisen auszugleichen. Hinzu kommen Abgaben aus der Produktion und den Lagerkosten von Zucker und Isoglukose, die von den Zuckerunternehmen zur Finanzierung der Erstattungen entrichtet werden, die zur Ausfuhr der über den EU-Bedarl hinausgehenden Zuckerproduktion gezahlt werden. Zölle werden vor allem im Rahmen des Gemeinsamen Zolltarifs erhoben. Bei den Mehrwertsteuermitteln handelt es sich um den an die Union abzuführenden Anteil der Mehrwertsteuereinnahmen der Mitgliedstaaten, der sich aus der Anwendung eines einheitlichen Satzes auf eine von Gemeinschaftsvorschriften bestimmte einheilliche Bemessungsgrundlage ergibt. Diese Bemessungsgrundlage ist für alle Mitgliedstaaten auf $50 \%$ des jeweiligen Bruttosozialprodukts begrenzt, für Großbritannien gilı (noch) ein besonderer Korrekturmechanismus. "' Bruttosozialproduktmittel schlieBlich sind solche Einnahmen, die sich am Bruttosozialprodukt der Mitgliedstaaten orientieren. Sie sind nicht an eine bestimmte Art von Staatseinnahmen geknüpft, sondern aus dem Staatshaushalt insgesamt zu erbringen. Die Einnahmen der Union aus Bruttosozialproduktmitteln sind derzeit auf 1,27\% des Bruttosozialprodukts der EU gedeckelt."

Auch der zur Zeit in der politischen Diskussion befindliche Eigenmittelbeschluss, der noch auf Art. 269 EG gestützı werden wird, beschränkt sich auf diese Eigenmit-

8 Waldhoff, in: Calliess/Ruffert (Hrsg.), EUV/EGV (Fn. 7), Art. 269 Rn. 3;

9 Vgl. Fischer, Der Europäische Verfassungsvertrag. 2005, S. 208 ff.

10 Vgl. An. 4 Beschluss 2000/597 über das System der Eigenmittel der Europäischen Gemeinschaften vom 29.09.2000. ABl. EG 2000 Nr. L. 253/42.

$11 \mathrm{Zu}$ den Einnahmearten und ihren Besonderheiten vgl. Niedobitek, in: Streinz (Hrsg.), EUV/EGV, 2003, Art. 269. 
telarten. Dagegen ermöglicht Art. I-54 Abs. 3 S. 2 VVE ausdrücklich. neue Kategorien von Eigenmitteln einzuführen und bestehende abzuschaffen. Diese Befugnis ersıreckt sich - theoretisch - auch auf die Möglichkeit einer Kreditfinanzierung. ${ }^{12}$ denn der Betonung der vollständigen Finanzierung aus Eigenmitteln in Art. I-54 Abs. 2 S. 1 VVE lässt sich kein absolutes Verbot einer Kreditfinanzierung entnehmen. Sie indiziert vielmehr lediglich, dass nur solche Einnahmen in den Haushalt nießen dürfen, die im Verfahren nach Abs. 3 festgelegt wurden. Ausgenommen sind nur ,sonstige Einnahmen“, zu denen vor allem die direkten Steuern auf die Bezüge der Bediensteten der Union sowie Verwaltungseinnahmen aus Gebühren, Zwangsgeldern, Säumnis-Zinsen und (kartellrechtlichen) Geldbußen zählen. ${ }^{1.3}$

\section{Höhe der Eigenmittel}

Zur Höhe der Eigenmittel bestimmt Art. I-54 Abs. 1 VVE in Übernahme der Regelung des Art. 6 Abs. 4 EUV, dass sich die Union mit den erforderlichen Mitteln ausstattet, um ihre Ziele erreichen und ihre Politik durchführen zu können. Diese Bestimmung geht auf einen politischen Kompromiss um die Finanzierung des Kohäsionsfonds vor Unterzeichnung des Maastricht-Vertrags zurück, der in seiner Bedeutung aber immer undeutlich geblieben ist. ${ }^{14}$ Umstritten war zum einen der Begriff "Mittel" und zum anderen die rechtliche Bedeutung der Bestimmung. Durch ihre neue systematische Stellung im Zusammenhang mit den Einnahmen ist nun jedenfalls klar, dass mit Mitteln nur finanzielle Mittel ${ }^{15}$ und jedenfalls keine Kompetenzen gemeint sind: Art. I-54 Abs. I VVE begründel keine Kompetenz-Kompetenz. ${ }^{16}$

Doch auch unter der Beschränkung auf finanzielle Mittel lassen sich Art. I-54 Abs. 1 VVE allenfalls mittelbar rechtliche Gehalte entnehmen. Keinesfalls lässt die reflexive Struktur des Satzes darauf schließen, dass sich die Union (eigenmächtig) mit finanziellen Mittel ausstattet. Über die Einnahmen wird ausschließlich in dem von Art. I-54 Abs. 3 VVE beschriebenen Verfahren entschieden. Immerhin jedoch hebı die Bestimmung hervor, dass der Union jedenfalls die Einnahmen zur Verfügung stehen müssen, die sie zur Finanzierung der ihr übertragenen Aufgaben benötigt. Insofern deutet Abs. 1 eine Konnexität zwischen den Aufgaben und den Ausgaben an, die wegen des Grundsatzes des Haushaltsausgleichs auch die Einnahmen erfasst. Somit laisst sich der Bestimmung in Konkretisierung des Grundsatzes der loyalen Zusammenarbeil (Art. I-5 Abs. 2 VVE) jedenfalls die politische Verpflichtung der Mitgliedstaaten entnehmen. der Union in dem Verfahren nach Art. I-54 Abs. 3 VVE Eigenmittel in einer Höhe zu bewilligen, die eine ordnungsgemäße

12 Zur Kreditfinanzierung der Union vgl. Waldhoff. in: Calliess/Ruffert (Fn. 7), Art. 269 Rn. 14 ff.

13 Näher Bieber, in: Groeben/Schwarze (Hrsg.), EU-/EG-Vertrag. 6. Aufl. 2004. Art. 269 Rn. 36 ff.

14 Vgl. Puttler, in: Calliess/Ruffert (Hrsg.). EUV/EGV. 2. Aun. 2002. Art. 6 EUV Rn. 219 ff.

15 Vgl. BR.Drs. 983/04, S. 267.

$16 \mathrm{Vgl}$. BVerfGE 89, 55, $195 \mathrm{f}$. 
Aufgabenerfüllung erlaubt. ${ }^{17}$ Eine rechrliche Durchbrechung des die Haushaltsverfassung prägenden Grundsatzes des Einnahmenhaushalts bewirkt die Bestimmung dagegen nicht.

\section{Grundsatz des Einnahmenhaushalts}

Nach diesem Grundsatz determinieren die der Union zur Verfügung stehenden Einnahmen und nicht etwa die ihr übertragenen Aufgaben die Ausgaben der Union. Die Festschreibung dieses Grundsatzes war den Mitgliedstaaten offensichtlich besonders wichtig, denn er kommt in verschiedenen Verfassungsbestimmungen zum Ausdruck. So verpflichtet Art. III-404 Abs. 10 VVE die am Haushaltsverfahren beteiligten Organe, ihre Haushaltsbefugnisse stets unter Beachtung der Eigenmittelgeselze auszuüben; gleiches gilt gemäB Art. III-405 Abs. 2 S. 1 VVE auch für das Nothaushaltsrecht. Auch die in Art. I-53 VVE verankerten Haushaltsgrundsätze der sachlichen Rechtsgrundlage (Abs. 4) und der Haushaltsdisziplin (Abs. 5) dienen der Sichenung des Grundsatzes des Einnahmenhaushalts. Vor allem aber bestimmt Art. I55 VVE für die Finanzrahmengesetze in Abs. 1 ausdrücklich, dass sich die Entwicklung der Ausgaben in den Grenzen der Eigenmittel halten muss, und erstreckt diese Verpflichtung in Abs. 3 auch auf die jährlichen Haushaltsgesetze.

\section{Mehrjähriger Finanzrahmen}

Ebenfalls ein Novum im VVE ist die in Art. I-55 und Art. III-402 erfolgte Konstitutionalisierung der Bestimmungen über den mehrjährigen Finanzrahmen. Sie waren bislang lediglich Gegenstand Interinstitutioneller Vereinbarungen.

\section{Funktion und Inhalt der Finanzrahmengesetze}

Der mehrjährige Finanzrahmen stellt ein Bindeglied zwischen den im Eigenmittelgesetz festgelegten Einnahmen und den in den jührlichen Haushaltsgesetzen vorgesehenen Ausgaben dar. Er soll als mittelfristige Finanzplanung einerseits eine geordnete Entwicklung der Ausgaben ermöglichen, andererseits aber vor allem auch sicherstellen, dass die Summe der Ausgaben der Union die Höhe der im Eigenmittelgesetz vorgesehenen Einnahmen nicht überschreitet. $\mathrm{Zu}$ diesem $\mathrm{Zweck}$ werden gemäß Art. I-55 Abs. 1 S. 2 VVE die Obergrenzen der Verpflichtungsermächtigungen für mindestens fünf Jahre im voraus (Art. Ill-402 Abs. I VVE) nach wenigen, den Haupttiatigkeitsbereichen der Union entsprechenden Ausgabenkategorien in einem Europäischen Gesetz des Rates festgesetzt (Art. III-402 Abs. 2 S. 2 VVE). Mit der gebotenen Gliederung der Verpflichtungsermächtigungen in Ausgabekategorien und deren Kongruenz zu den Haupttaitigkeitsbereichen der Union soll die

17 Ohne Bezug auf Ar. I-54 Abs. 1 (bzw. An. 6 Abs. 4 EUV), aher im Ergebnis ähnlich Bieber, in: Grocben/Schwarze (Fn. 13), Vorbem. An. 268 - 280 Rn. 6; Magiera, in: Grabilz/Hilf (Hrsg.), Das Recht der EU, Maastrichter Fassung, Art. 201 Rn. 11. 
Ühersichllichkeit, mit der Beschränkung auf wenige Ausgabenkategorien die Flexibilität des mehrjährigen Finanzrahmens gewährleistet werden. ${ }^{18}$

\section{Bedeutung der Finanzrahmengesetze}

Die besondere Bedeutung dieser Finanzrahmengesetze liegt darin, dass sie die Haushaltshehörde gemäß Art. I-55 Abs. 3 VVE bei der Aufstellung der jährlichen Haushaltspläne binden. Die Konstitutionalisierung der mittelfristigen Finanzplanung führt insofern zu einer verfassungsrechllich vorgesehenen Hierarchie der Finanzbestimmungen: Die durch Eigenmittelgesetz festgelegten Einnahmen der Union begrenzen die im Finanzrahmengesetz. mittelfristig veranschlagten Ausgaben der Union, die ihrerseits bei der Aufstellung der Jahreshaushaltspläne zu beachten sind. Diese Hierarchie in den Finanzbestimmungen spiegelt zugleich die Machtverteilung zwischen den Mitgliedstaaten und der Union wieder: Während die Eigenmittelgesetze nicht nur der einstimmigen Entscheidung des Rates, sondern zudem der Ratifizierung durch die Mitgliedstaaten bedürfen und somit noch dem primüren Recht zuzurechnen sind, fällt der Erlass der Finanzrahmengesetze zwar schon vollständig in die Kompetenz der Union und somit in den Bereich sekundärer Rechtsetzung, verlangt aber wegen der besonderen politischen Bedeutung immer noch die Einstimmigkeit im Rat. Erst die jährlichen Haushaltsgesetze können mit qualifizierter Mehrhejt des Rates erlassen werden.

Die Festlegung der miltelfristigen Finanzplanung erschien den Mitgliedstaaten (insbesondere den Nettozahlerstaaten) so wichtig, dass sie die von Art. 1-54 Abs. 4 VVE/KonvE vorgesehene Regelung noch einmal modifizierten. Nach dieser Regelung sollte nur der erste mehrjährige Finanzrahmen nach lnkrafttreten der Verfassung einstimmig. die nachfolgenden konnten dagegen mil qualifizierter Mehrheit festgelegt werden. Nunmehr enthält Art. I-55 Abs. 4 VVE lediglich (aber immerhin) eine Passerelle-Klausel, nach der der Europäische Rat einstimmig den Ühergang zur qualifizierten Mehrheit beschließen kann. Ohne diese Klausel bedürfte der Wechsel in den Mehrheitserfordernissen eines Vertragsänderungsverfahrens nach Art. IV-443 VVE. ${ }^{19}$

Dass die Verabschiedung der Finanzrahmengesetze auch künftig schwierig sein wird, weil es bei den Obergrenzen der Ausgaben für die ..Haupttätigkeitsbereiche“ der Union letztlich um die Frage geht. wie viele Mittel der Union in die einzelnen Mitgliedstaaten zurückfließen, hat auch der VVE erkannt. Er bestimmt deshalb in Art. III-402 Abs. 4 VVE (funktional dem Nothaushaltsrecht ähnelnd), dass für den

18 Eine konkrete Anzahl lässt sich der Bestimmung nicht entnehmen. Bislang wurden acht Rubriken unterschieden. die Interinstitutionelle Vereinbarung 2007.2013 sicht sechs. ihr Entwurf sah nur vier Rubriken vor.

19 Die Niederlande haben in ihrer einseitigen Erklärung Nr. 42 zur Schlussakte erklär, dass sie dem Übergang in die qualifizierte Mehrheit zustimmen werden. ..sobald im Rahmen der Überprüfung des Eigenmittelgesetzes für die Niederlande eine zufriedenstellende Lösung für ihre [...] äußersı nachteilige Position als Nettozahler gefunden wurde.“ 
Fall, in dem der Rat bis zum Ablauf des geltenden Finanzrahmengesetzes kein neues Finanzrahmengesetz erlassen hat, die Obergrenzen des letzten Jahres des laufenden Finanzrahmens zu beachten sind.

\section{Haushaltsgrundsärze}

Art. I-53 VVE fasst einige Haushalts- und Finanzgrundsätze zusammen, die im gesamten Haushaltskreislauf der Union von der Festlegung des Haushaltsplans über dessen Ausführung bis hin zur Entlastung der Kommission zu beachten sind. Im EG-Vertrag waren sie noch in unterschiedlichen Bestimmungen verankert. Im Einzelnen lassen sich der Norm entnehmen: Grundsatz der Vollständigkeit und Grundsatz der Einheit (Abs. 1), Grundsatz des Haushaltsausgleichs (Abs. 2), Grundsatz der Jährlichkeit (Abs. 3), Grundsatz der Haushaltsdisziplin (Abs. 5), Grundsatz der Wirtschaftlichkeit (Abs. 6). Das in Abs. 4 erstmals normiente Erfordernis einer sachlichen Rechtsgrundlage für die Mittelausführung dürfte eher als Finanzgrundsalz zu qualifizieren sein, ohne dass eine exakte terminologische Unterscheidung zwischen Haushalts- und Finanzgrundsätzen möglich oder geboten wäre. Gleiches gilt für den in Abs. 7 angesprochenen Schutz der finanziellen Interessen der Union, der an dieser Stelle nur im Grundsatz betont und im Übrigen durch Art. III-415 VVE konkretisiert wird.

Weitere Haushaltsgrundsäize finden sich im Dritten Teil des VVE (Grundsatz der Spezialität - Art. III-407 VVE; Grundsatz der Rechnungseinheit - Art. IIl-410 VVE) oder können in der Haushaltsordnung niedergelegt werden (bspw. Grundsatz der Gesamtdeckung, Bruttoprinzip u.a.).

\section{Bedeulung der Haushaltsgrundsätze}

Die Bedeutung der von Art. I-53 VVE hervorgehobenen Haushalts- und Finanzgrundsätze erklärt sich vor allem mit Blick auf die Funktionen, die dem Haushaltsplan der EU zukommen. Mit der Finanzwissenschaft lassen sich vier Funktionen öffentlicher Haushalte unterscheiden: ${ }^{20}$ Die finanzpolitische Funktion besteht in der Veranschlagung der zur Ausgabendeckung erforderlichen Finanzmittel. Die politische Programmfunktion kommt in den entsprechend dem Grundsatz der Spezialität vorzunehmenden Festsetzungen der Mittel nach Höhe und Zweck zum Ausdruck: Der Haushaltsplan ist stets ein politisches Handlungsprogramm in Zahlen. Die Kontrollfunktion des Haushaltsplans basiert auf der rechtlichen Bindung der ihn vollziehenden Organe, insbesondere also der Kommission, an die in ihm enthaltenen Festsetzungen: Sie ermöglicht die Überprüfung der zahlenmïßigen und rechnerischen Richtigkeit und Rechtmaißigkeit ebenso wie der Wirtschaftlichkeit der Haushaltsführung. Die wirtschafispolitische Funkrion, die bei nationalen Haushalten darin besteht, dass diese auf Grund ihres Volumens erheblichen Einfluss auf die allgemeine

$20 \mathrm{Vgl}$. für das deutsche Recht Kisker, in: Isensee/Kirchhof (Hrsg.). HStR IV, 1990, § 89 Rn. 13; für die EU vgl. Bieber, in: Groeben/Schwarze (Fn. 13), Vorbem. Art. 268 Rn. 19 f.; Waldhoff, in: Calliess/Ruffert (Fn. 7), Ant. 268 Rn. 9. 
konjunkturelle Entwicklung nehmen können, steht bei dem Haushalt der EU im Hintergrund: Zu gering ist sein Volumen, um die gesamtwirtschaftliche Entwicklung maßgeblich heeinflussen zu können. ${ }^{21}$ Gleichwohl sind Teilbereiche des EUHaushalts, etwa im Agrarbereich, sowie die verschiedenen vom VVE vorgesehenen und im Haushalt ausgewiesenen Umverteilungen zwischen wohlhabenderen und weniger wohlhabenden Mitgliedstaaten geeignet, der gesamtwirtschaftlichen Entwicklung vereinzelte Impulse $z$ geben. ${ }^{22}$

Zur Gewährleistung dieser Funktionen haben sich Haushaltsgrundsätze herausentwickelt, die als Maßstab für die Haushaltsaufstellung, -ausführung und -kontrolle dienen. Rechtliche Bedeutung erlangen sie regelmäßig erst durch ausdrückliche Normierung, wenngleich sie im Einzelfall auch aus allgemeinen Rechtsgrundsätzen abgeleitet werden mögen. Doch auch bei normativer Verankerung wie in Ar. I-53 VVE bleiben sie als Grundsätze offen für Ausnahmen und Durchbrechungen, die zum Teil schon im VVE selbst angeordnet werden. Ihre konkrete Ausgestalıung ist darüber hinaus der nach Art. 1-56 VVE zu erlassenden Haushaltsordnung und somit dem einfachen europiischen Gesetzgeber vorbehalten. Den verfassungsrechtlichen, der Ausgestaltung durch die Haushaltsordnung entzogenen Kern der Haushaltsgrundsätze zu bestimmen, wird im Einzelfall sehr schwierig sein. Gleichwohl ist ihre ausdrückliche Normierung im ersten Teil des VVE nicht ohne Bedeutung: Sie signalisiert, dass etwaige von der Haushaltsordnung vorgesehene Ausnahmen der (rechtlichen) Rechtfertigung durch einen sachlichen Grund bedïrfen. Darüber hinaus wirken die Haushaltsgrundsätze als politischer Maßstab sowohl auf den Erlass der Haushaltsordnung als vor allem auch auf die Festlegung und die Ausführung der jährlichen Haushaltspläne ein: Abweichungen von den Grundsätzen sind vor allem auch politisch zu verantworten. ${ }^{23}$

\section{2. Änderungen gegenüber dem EG-Vertrag im Überblick}

Neben kleineren Modifizierungen sind insbesondere fünf Unterschiede zwischen Art. I-53 VVE und den entsprechenden Bestimmungen im EG-Vertrag hervorzuheben: Allgemein spricht Abs. I nun vom Haushalısplan der Union und differenziert insoweit nicht mehr zwischen den Einnahmen und Ausgahen der Gemeinschaft auf der einen Seite und den Ausgaben der Unionspolitiken auf der anderen Seite. Daruber hinaus tragen Abs. 5 Halbs. 1 und Abs. 6 dem Umstand Rechnung. dass der Haushaltsplan zwar vorrangig, aber nicht nur von der Kommission ausgeführt wird:

21 Der Haushalt 2005 macht mit einem Volumen von 106 Mrd. Euro etwa 1\% des Bruttonationaleinkommens der 25 Mitgliedstaaten aus.

22 Zum Finanzausgleich s. Lienemeyer, Die Finanzverfassung der Europäischen Union. 2002, S. $262 \mathrm{ff}$.

23 Zur allgemeinen Bedeutung der Haushaltsgrundsätze vgl. Stem. Staatsrecht II. S. 1236 ff: Kisker. in: Isensee/Kirchhof (Fn. 20). \$ 89; zur Bedeutung der Grundsäıze in der EU vgl. Rossi. Europäisches Parlament und Haushaltsverfassungsrecht. 1997, S. 177 ff.: Waldhoff, in: Calliess/Ruffert (Fn. 7), Art. 268 Rn.19; Bieber, in: Groeben/Schwarze (Hrsg.). EU-/EG-Vertrag (Fn. 13). Ar. 268 Rn. 2. 
Die Deckungsnotwendigkeit für Ausgaben erstreckt sjch deshalb auch auf die anderen Organe der Union, die zudem ausdrücklich auch vom Grundsatz der Wirtschalilichkeit verpflichtet werden. Weiterhin hestimmt Abs. 4 nunmehr explizit, dass die Ausführung der in den Haushaltsplan eingestellten Ausgaben grundsäitzlich einen verbindlichen (sachlichen) Rechtsakt der Union voraussetzt, und Abs. 5 Halbs. 2 betont in Ergänzung des Art. I-55 Abs. 3 VVE, dass die Mittelansätze im mehrjährigen Finanzrahmen nicht nur vom Haushaltsgesetzgeber, sondern auch vom sachlichen Gesetzgeber als Obergrenze zu beachten sind.

\section{Grundsatz der sachlichen Rechtsgrundlage}

Nähere Beachtung verdient die gegenüber dem EG-Vertrag neue Regelung Art. I-53 Abs. 4 VVE, nach der die Ausführung der im Haushalısplan veranschlagten Ausgaben grundsätzlich eines verbindlichen (sachlichen) Rechtsakts der Union bedarf. ${ }^{24}$ Der Haushaltsansatz ist somit regelmäßig nur notwendige, nicht aber hinreichende Bedingung für die Ausgabe von Gemeinschaftsmitteln. Dies ist zwar seit 1990 durch eine entsprechende Bestimmung in der Haushaltsordnung ${ }^{25}$ anerkannt und vom EuGH 1998 zumindest für „bedeutende Gemeinschaftsaktionen“ auch deutlich hervorgehoben worden, ${ }^{26}$ war in der Vergangenheit gleichwohl immer wieder streitig. ${ }^{27}$ Auch Abs. 4 löst die Frage nur im Grundsalz und erlaubı es der Haushaltsordnung, Ausnahmen von diesem Grundsalz vorzusehen. Dabei mag auch die Frage geklairt werden, ob der Rat in Rechtsakten eine Mitentscheidung von Ausschüssen über die Verwendung bewilligter Mittel vorsehen darf. ${ }^{28}$

Mit der durch den VVE bewirkten zunehmenden (noch immer aber nichı vollständigen) Kongruenz zwischen den haushaltsrechtlichen und den sachlichrechtlichen Befugnissen des Parlaments verliert die Normierung des Grundsatzes der sachlichen Rechtsgrundlage zwar ein wenig an Brisanz, bleibt aber für die Kompetenzabgrenzung zwischen Rat und Parlament als primäre Legislativorgane auf der einen Seite und der Kommission als vorrangiger Exekutivbehörde aul der anderen Seite von Bedeutung. Wenn darüber hinaus auch Rechte Dritter betrolfen sind, indiziert der Grundsatz zugleich die Reichweite des rechtsstaatlichen Gesetzesvorbehalts.

24 Magiera (Fn. 3), S. 625. spricht insofern von der Notwendigkeit eines „Basisrechtsakts“. Ebenso Waldhoff, in: Calliess/Ruffert (Hrsg.). VerfEU, Art. I-53 Rn. 23.

25 Art. 49 HO 2002 (VO 1605/2002) vom 25.06.2002, ABI. EG 2002 Nr. L 248/1, spricht insofern von ,.Basisrechtsakten“.

26 EuGH, Rs. C-106/96. Vereinigtes Königreich/Kommission. Slg. 1998, S. 2729; kritisch hierzu Bieber, in: Groeben/Schwarze (Fn. 13), Ant. 274 Rn.ll ff.

27 Zsfd. Bieber, in: Groeben/Schwarze (Fn. 13), Art. 274 Rn. 11 ff.; Waldhoff, in: Calliess/Ruffen (Fn. 7). Arr. 274 Rn. 3: vgl. Rossi, Europiaisches Parlament und Haushaltsverfassungsrecht (Fn. 23) S. 128 ff.; Ausnalumen vom Grundsalz der sachlichen Rechtsgrundlage sieht Nr. F 37 der Interinstitutionellen Vereinbarung vom 6.05.1999, ABI. EG 1999 Nr. C 172/11, vor.

28 Vgl. hierzu EuGH, Rs. 16/88, Kommission/Rat, Slg. 1989, S. 3457, sowie Schoo, in: Schwarze (Hrsg.), EU-Kommentar, Art. 274 Rn. 9; Magiera, in: Grabitz/Hilf (Fn. 17), Art. 205 Rn. 4; Niedobitek, in: Sireinz (Fn. 11), Art 274 Rn. 4. 


\section{Grundsatz der Haushaltsdisziplin}

Der Grundsatz der Haushaltsdisziplin bedeutet, dass bei der ausgabenwirksamen Rechtsetzung der Union stets die jeweils verfügbaren Eigenmittel zu berücksichtigen sind. Der Grundsatz ist mit dem durch den Wechsel von der Beitrags- zur Eigenmittelfinanzierung bedingten Wandel von einem Ausgaben- zu einem Einnahmenhaushalt entstanden. Seine besondere Relevanz erhält er vor allem dadurch, dass der Haushalt der Union nach Maßgabe der bisherigen Eigenmittelbeschlüsse grundsätzlich nicht über Kredite finanziert werden darf. Konkretisient wird der Grundsatz derzeit durch eine Interinstitutionelle Vereinbarung ${ }^{29}$ sowie eine Verordnung des Rates. ${ }^{30}$

Der Anwendungsbereich des Grundsatzes beschränkt sich auf Rechtsakte mit erheblichen Auswirkungen auf den Haushaltsplan und somit auf solche. die die im Haushaltskreislauf üblicherweise auftretenden Schwankungen deullich übersteigen." Adressat des Grundsatzes ist nicht - wie nach Art. 270 EG - allein die Kommission, sondern die Union. Sämtlichen ihrer Organe, insbesondere dem Parlament und dem Rat. ist es deshalb verboten. ausgabenwirksame Rechtshandlungen vorzunehmen, die nicht finanziert werden können. Der dabei anzuwendende Maßstab ist ein doppelter: Die mit den Rechtsakten verbundenen Ausgaben müssen sowohl mit den veranschlagten Eigenmittelı der Union als auch mit den Vorgaben des mehrjährigen Finanzrahmens im Einklang stehen. Für den Fall, dass die Eigenmiltel ausnahmsweise die Mittelansätze des mehrjährigen Finanzrahmens übersteigen, erlangt dieser dadurch über Art. I-53 Abs. 3 VVE hinaus auch für den sachlichen Geselzgeber an Bedeutung.

Wegen der binnengerichteten Zielsetzung des Grundsatzes soll sich seine Verletzung nur im Verhältnis zwischen den Organen auswirken, nicht jedoch zur Rechiswidrigkeit der beschlossenen Rechtsakte führen. ${ }^{32}$ Diese Beschränkung führt jedoch nicht zu dem für die Gewährleistung der Haushaltsdisziplin notwendigen Sanktionsdruck und ist deshalb aufzugeben. Schäden Dritter sind gegebenenfalls nach den Haftungsregelungen des Art. III-43I VVE zu beseitigen.

\section{Haushalispläne}

Von zentraler Bedeutung für die Durchführung der einzelnen Politikbereiche bleiben die Haushaltspläne. Sie allein ermächtigen die ausführenden Organe, die von ihm bewilligten Mittel zweckentsprechend zu verwenden (Art. III-407 UA 1 S. I VVE).

29 Interinstitutionelle Vereinbarung $2007-2013$ vom 14.06.2006. ABI. $2006 \mathrm{Nr}$. C $139 / 1$.

30 VO 2040/2000 v. 26.09.2(x)(. ABI. EG 2000 Nr. L 244/27.

31 Waldhoff. in: Calliess/Ruffen (Fn. 7), Ar. 27 I Rn. 3.

32 Bieber, in: Groeben/Schwarze (Fn. 13). Art. 270 Rn. 6. 


\section{Der Haushaltsplan als Europäisches Binnengesetz}

Rechtliche Verbindlichkeit erhält der Haushaltsplan erst durch seine Feststellung in dem dafür vorgesehenen Verfahren. Während die Rechtsnatur dieser Feststellung vom EG nicht eindeutig definiert war, bestimmt Art. I-56 VVE nunmehr, dass der Haushaltsplan durch und d.h. als Europäisches Gesetz aufgestellt wird. Praktisch wird er dem Feststellungsgesetz als Anhang beigefügt und bildet mit ihm zusammen eine Einheit.

Trotz seiner Bezeichnung als Europäisches Gesetz unterscheidet sich der Haushaltsplan in seinen Rechtswirkungen von denjenigen Europäischer Gesetze im Sinne des Art. 1-33 Abs. 1 UA 2 VVE: Die Kommission und die anderen Organe der Union werden durch den Haushaltsplan ermächtigt, die in ihm vorgesehenen Mittel (vorbehaltlich einer entsprechenden sachlichen Rechtsgrundlage - Art. I-53 Abs. 4 VVE) zu verwenden; die Mitgliedstaaten werden verpflichtet, die geschuldeten Anteile an den Mehrwertsteuereinnahmen und Bruttosozialproduktmitteln bereitzustellen. Dagegen werden private Dritte vom Haushaltsplan weder berechtigt noch verpflichtet. Wegen dieser auf die Hoheitsträger im Verbund der Union und der Mitgliedstaaten beschränkten Rechtswirkungen ist der Haushaltsplan als bloßes Binnengesetz zu qualifizieren.

\section{Festlegung des Haushaltsplans}

Die Festlegung des Haushaltsplans als Europäisches Gesetz bringt die Intention des VVE zum Ausdruck, das Haushaltsverfahren dem regulären Gesetzgebungsverfahren anzugleichen. Zugleich ordnet Art. I-56 VVE jedoch an, dass der Haushaltsplan nicht in dem Verfahren nach Art. III-396 VVE, sondern in einem besonderen, von Art. III-404 VVE vorgesehenen Verfahren erlassen wird, das sich immer noch deutlich von dem regulären Gesetzgebungsverfahren unterscheidet. ${ }^{33}$

\section{a) Phasen der Haushaltsfestlegung}

Das von Art. III-404 VVE normierte Haushaltsverfahren sieht bis zu sechs Phasen vor. Sämtliche Phasen sind durch strenge Fristen gekennzeichnet, die gewährleisten sollen, dass der Haushaltsplan entsprechend dem Grundsatz der Vorherigkeit regelmäßig vor Beginn des Haushaltsjahres (das gemäß Art. III-403 VVE dem Kalenderjahr entspricht) festgelegt wird. Sicherstellen können sie dies allerdings nicht: Bei Ausnutzung aller Verfahrensrechte und -fristen kann der Haushaltsplan nicht bis zum 1. Januar festgelegt werden. In diesem Fall tritt automatisch das von Art. III405 VVE geregelte Nothaushaltsrecht in Kraft. Um die damit einhergehenden Beschränkungen für die Haushaltsausführung zu vermeiden, werden sich die am Haus-

33 Unbefriedigend ist dabei die unterschiedliche Terminologie: Während Art. 272 EGV bislang die Feststellung des Haushaltsplans regelte, spricht Ar. I-56 nun von seiner Aufstellung und Art. III-404 von seiner Festlegung. Mangels erkennbarer rechllicher Unterschiede dieser Begrifflichkeiten ist davon auszugehen, dass sie synonym verwendet werden. 
haltsverfahren beteiligten Organe möglicherweise wieder auf einen pragmalischen Kalender einigen, der kürzere Fristen und frühere Fixpunkte vorsieht. ${ }^{3 . t}$

Die Haushaltsfestlegung beginnt mit der Vorbereitungsphase, in der die Organe nach Maßgabe interner Vorschriften Voranschläge für ihre Ausgaben des nächsten Haushaltsjahres aufstellen. Sie werden von der Kommission zu einem Haushaltsplanentwurf zusammengefasst, der auch einen vollständigen Überblick über die Einnahmen enthalten muss. Ein Vorentwurf, wie er von Art. 272 Abs. 3 EG vorgesehen ist, wird nicht erstellt.

In der anschließenden Berafungsphase diskutieren Rat und Parlament den von der Kommission aufgestellten Haushaltsentwurf getrennt in jeweils einer Lesung und schlagen gegebenenfalls Änderungen vor. Ihre Änderungsrechte divergieren nicht nach obligatorischen und nicht-obligatorischen Ausgaben, sondern erstrecken sich gleichermaßen auf alle Ausgaben. Nachdem zunächst der Rat detailliert begründete Standpunkte zu dem Entwurf vorzulegen hat, über die er mangels abweichender Bestimmungen gemäß Art. I-23 Abs. 3 VVE mit qualifizierter Mehrheit i.S.v. Art. I25 VVE entscheidet, hat sodann das Parlament drei Reaktionsmöglichkeiten: Billigt es den Standpunkt des Rates (Abs. 4 lit. a) oder fasst es innerhalb von 42 Tagen nach der Übermittlung keinen Beschluss (Abs. 4 lit. b), gilt der Haushaltsplan als erlassen. In diesen Fällen wandelt sich die Beratungsphase zur Entscheidungsphase. Nimmt es dagegen Änderungen an dem Entwurf vor (Abs. 4 lit. c), wird die geänderte Fassung zurück an den Rat und die Kommission geleitet. Hervorzuheben ist, dass Änderungen nur mit absoluter Mehrheit des Parlaments beschlossen werden können, während für die Billigung der Standpunkte des Rates mangels abweichender Regelung gemäß Art. III-338 VVE die relative Mehrheit ausreichend ist. Sofern das Parlament Änderungen am Entwurf beschließt, kanı der Rat diese Änderungen binnen zehn Tagen (mit qualifizierter Mehrheit) billigen und den Haushaltsplan damit inhaltlich festlegen. Auch für diesen Fall erweist sich die Beratungs- als Entscheidungsphase, der nur noch die Feststellungsphase zu folgen hat. Allerdings düfte es in der Praxis jedenfalls nicht beim ersten Durchlauf eines Kommissionsentwurfs zu einer frühzeitigen Festlegung des Haushaltsplans kommen. Nur wenn die Kommission nach fruchtlosem Ablauf des Vermittlungsverfahrens einen erneuten Vorschlag vorlegt. erscheint es vorstellbar, dass ein Teil der Haushaltsbehörde die Änderungen des jeweils anderen Teils bereits in dieser Phase billigt.

Sofern sich Rat und Parlament nicht über ihre Änderungsvorschläge einigen, tritt in der Verminlungsphase ein Vermittlungsausschuss zusammen. Seine Zusammensetzung und seine Arbeitsweise bedürfen der näheren Ausgestaltung, bspw. in der nach Art. III-412 VVE zu erlassenden Haushaltsordnung. Dabei sind die verfassungsrechtlichen Vorgaben des Art. III-404 Abs. 5 VVE zu wahren und insbesondere zu beachten, dass der Vermittlungsausschuss auf der Grundlage der Standpunkte von Rats und Parlament tätig wird und insofern keine grundsätzlich anderen Positio- 
nen in den Einigungsprozess einbringen darf. Die Kommission nimmt an den Sitzungen des Vermittlungsausschusses teil, ist dabei aber auf eine informative und mediative Rolle beschränkt und darf ihre Beteiligung nicht dazu missbrauchen, ihren eigenen Entwurf grundlegend zu revidieren. Einigt sich der Vermittlungsausschuss binnen 21 Tagen nicht auf einen gemeinsamen Entwurf, ist die Kommission zur Vorlage eines neuen Entwurfs verpflichtet (Art. III-404 Abs. 9 VVE), an die sich eine neue Beratungsphase anschließt. Einigt er sich, beginnt die Entscheidungsphase (Art. III-404 Abs. 6 VVE).

In dieser Entscheidungsphase müssen grundsätzlich beide Organe den gemeinsamen Entwurf billigen, was ihre Gleichberechtigung bezüglich aller Ausgaben zum Ausdruck bringt.

Allerdings hat das Parlament in einer Konstellation die Möglichkeit, in einer anschließenden Bestätigungsphase alle oder einige der von ihm zunächst mit absoluter Mehrheit vorgenommenen Abänderungen des Kommissionsentwurfs nach der Einschaltung des Vermittlungsausschusses binnen 14 Tagen mit einer qualifizierten Mehrheit, der sog. Haushaltsmehrheit, zu bestätigen. Diese Mehrheit entscheidet über den Inhalt des Haushaltsplans: Wird sie erreicht, wird der entsprechende Haushaltsposten nach Maßgabe der Änderungen des Parlaments festgelegt, wird sie verfehlt, ist der im Vermittlungsausschuss vereinbarte Standpunkt zu diesem Haushaltsposten maßgeblich. Dies ist das politische Risiko, welches das Parlament eingeht, wenn es versucht, in der Bestatigungsphase das letzte Wort über den Haushalt zu erhalten.

Abgeschlossen wird das Haushaltsverfahren erst in der Feststellungsphase durch die förmliche Erklärung des Parlamentspräsidenten, dass das Haushaltsgesetz endgültig erlassen ist. Diese Feststellung ist konstitutiver und nicht nur deklaratorischer Natur, sie erst führt zur Rechtswirksamkeit des Haushaltsplans. Dies gilt insbesondere, wie in der Formulierung „endgültig" zum Ausdruck kommt, auch für die Fälle, in denen Art. IIl-404 VVE die Festlegung des Haushaltsplans fingiert (..gilt als erlassen“). Diese Fiktion erstreckı sich insofern nur auf die inhaltliche Festlegung des Haushaltsplans, nicht jedoch auf seine rechtliche Wirksamkeit. ${ }^{35}$

\section{b) Beachtung der Einnahmen}

Neu ist die Vorschrift des Art. III-404 Abs. 10 VVE. Sie verpflichtet die am Haushaltsverfahren beteiligten Organe, bei der Ausübung ihrer Befugnisse die Verfassung sowie das auf ihrer Grundlage erlassene Recht zu wahren, und zielt damit auf die Beachtung des nach Art. 1-54 VVE erlassenen Eigenmittelsystems, der nach Art. 1-55 VVE festgelegten Finanziellen Vorausschau sowie der von Art. I-53 VVE normierten Grundsätze des Haushaltsausgleichs und der Haushaltsdisziplin. Sämtliche dieser Vorschriften dokumentieren den Grundsatz des Einnahmenhaushalts. Die am 
Haushaltsverfahren beteiligten Organe werden durch Abs. 10 somit dazu gemahnt, ihre Haushaltsbefugnisse nur soweit auszuüben, wie deren Resultate finanzierbar sind. Gegenüber den genannten speziellen Vorschriften kommt Art. III-404 Abs. 10 VVE im Wesentlichen eine affirmative Bedeutung zu.

\section{c) Priorität obligatorischer Ausgaben}

Von besonderer Bedeutung ist darüber hinaus auch Art. III-413 VVE. Er verpflichtet das Parlament, den Rat und die Kommission sicherzustellen, dass der Union Finanzmittel in einer Höhe zur Verfügung stehen, die es ihr ermöglichen, ihren rechtlichen Verpflichtungen gegenüber Dritten nachzukommen. Zwar lassen sich dieser Bestimmung für das Intraverbundverhältnis zwischen der Union und ihren Mitgliedstaaten keine rechtlichen Gehalte entnehmen. Die Art und die Höhe der der Union zur Verfügung stehenden Einnahmen bestimmen sich ausschließlich nach dem System der Eigenmittel, das im Verfahren nach Art. I-53 Abs. 3 VVE und somit letztlich von den Mitgliedstaaten beschlossen wird. Allenfalls lässt sich der Norm in Ergänzung des Art. I-53 Abs. 1 VVE das an die bezeichneten Organe gerichtete Gebot entnehmen, im Verfahren des Art. I-53 Abs. 3 VVE auf die Finanzierbarkeit der obligatorischen Ausgaben hinzuwirken. ${ }^{36}$

Dagegen erlangt die Bestimmung für das Interorganverhältnis zwischen den genannten Organen als Verteilungsgrundsatz erhebliche Bedeutung. Sie verpflichtet die Organe, die zur Verfügung stehenden Einnahmen im Haushaltsverfahren so zu bewilligen, dass jedenfalls die Finanzierung der obligatorischen Ausgaben gesichert ist. Andere Politiken können nur mit den verbleibenden Mitteln finanziert werden. Insofern beschränkt Ant. III-413 VVE die politische Gestaltungsfreiheit aller drei Organe. Das Parlament wird durch die Bestimmung im Vergleich zu seinen Befugnissen nach Art. 272 EG nicht einseitig benachteiligt, denn es hat im Haushaltsverfahren an Einfluss auf die obligatorischen Ausgaben gewonnen.

Im Außenverhältnis zu Dritten schließlich lässt sich Art. III-4I3 VVE derart als Haftungsregel verstehen, dass sich die Union nicht darauf berufen kann, die materiell begründeten Pflichten nicht finanzieren zu können. Als eigene Anspruchsgrundlage kommt die Norm aber nicht in Betracht, vielmehr bestimmen sich etwaige Ansprüche nach Art. III-43! VVE.

d) Würdigung der Haushaltsbefugnisse

Die mit der Ausgestaltung des Haushaltsverfihrens verbundene Verteilung der Haushaltsbefugnisse der einzeInen Organe, insbesondere des Parlaments. war bei der

36 Zur Dependenz zwischen Aufgaben, Ausgaben und Einnahmen s. Art. I-54 Rn. 4; Art. I-55 Rn. 8. 
Ausgestaltung des VVE bis zuletzt umstritten. ${ }^{37}$ Nach Art. Ill-404 VVE lassen sich die Haushaltsbefugnisse der Organe knapp wie folgt skizzieren:

Die Rolle der Kommission hat im Vergleich zur Regelung des Art. 272 EG an Bedeutung gewonnen. Hervorzuheben ist zunächst, dass die Kommission nach Art. III-404 VVE nicht nur einen Vorentwurf, sondern den Entwurf des Haushaltsplans ausarbeitet, dessen Erlass nach Art. 272 EG noch in die Zustaindigkeit des Rats fiel. Vor allem aber gewinnt die Kommission durch ihre - wenn auch nur informative und mediative - Beteiligung im Vermittlungsausschuss an zusätzlichen Einflussmöglichkeiten, die ihr nach Art. 272 EG formal nichı zustanden (die sie aber im Rahmen der von der Interinstitutionellen Vereinbarung vorgesehenen Konzertierungsverfahren und Triloge faktisch durchaus wahrgenommen hat).

Der Rat hat seine Entscheidungshoheit über die obligatorischen Ausgaben eingebüßt, dafür aber ein Mitentscheidungsrecht über die nicht-obligatorischen Ausgaben gewonnen. Außerdem muss er bei seinen Verhandlungen über den Haushalt nicht mehr befürchten, dass das Parlament den Haushalt als Ganzes ablehnt, und kann insofern offensiver auftreten. Die Möglichkeit, durch ein entsprechendes Stimmverhalten die Kommission zur Vorlage eines veränderten Haushaltsentwurfs zu veranlassen, dürfte die (politische) Position des Rates ebenfalls stärken.

Das Parlament kann erstmals auch über die obligatorischen Ausgaben entscheiden, hat dafür aber sein letztes Wort in Bezug auf die nicht-obligatorischen Aufgaben verloren. Für die politische Verhandlungsführung schwerer wiegt wohl der Verlust des Monopols, die Kommission durch eine Ablehnung des Haushalts aus wichtigen Gründen zur Vorlage eines neuen Entwurfs zu bewegen (Art. 272 Abs. 8 EG). Aufgewogen wird dieser Verlust jedenfalls teilweise durch die Möglichkeit, mit der Haushaltsmehrheit die eigenen politischen Vorstellungen durchsetzen zu können.

Gemessen an der Verteilung der Einnahmen- und Ausgabenkompetenzen und an der demokratischen Legitimation der Union sind die Haushaltsbefugnisse der Organe im Haushaltsverfahren adäquat verteilt. Ein umfassendes und alleiniges Budgetrecht des Parlaments passte nicht zu diesen Bedingungen. Denn wegen fehlender Einflussmöglichkeiten des Parlaments auf die Einnahmen besteht kein hinreichender Entscheidungszusammenhang zwischen den Einnahmen und den Ausgaben. Das bedeutet, dass das Parlament ausgabenwirksame Maßnahmen beschließen kann, ohne sich für die damit verbundenen finanziellen Belastungen gegenüber dem Wähler verantworten zu müssen. Solange also das Parlament keine Entscheidungsbefugnisse bezüglich der Einnahmen besitzt, stellı der Rat das einzige Verbindungsglied in der Verantwortungsbeziehung zwischen Einnahmen und Ausgaben dar. ${ }^{38}$

37 Vgl. Fische (Fn. 9). S. 485 f.

38 Näher Russi (Fn. 23), S. 246 ff. 


\section{Ausführung des Haushaltsplans}

Mit dem Erlass des Haushaltsgesetzes werden die im Haushaltsplan eingesetzten Mittel für ein Haushaltsjahr bewilligt (Art. I-53 Abs. 3 VVE). Die Ausführung des Haushaltsplans, die Verwendung der in ihn eingesetzten Mittel, obliegt nach Art. III407 UA I VVE neben der Kommission auch den anderen Organen der Union. Darüber hinaus sind - mangels eigenen Verwaltungsunterbaus der Union - auch die Mitgliedstaaten im indirekten Vollzug beteiligt, was Art. III-407 UA I S. I VVE nun noch deutlicher betont. Allerdings bleibt trotz der Erwähnung der Mitgliedstaaten allein die Kommission für die Ausführung des Haushaltsplans verantwortlich. Das folgt nicht nur aus einer exakten grammatikalischen Auslegung des Absatzes, sondern auch aus dem notwendigen Korrelat zwischen Verantwortlichkeit und Entlastung: Da die Entlastung nach Art. Ill-409 Abs. I S. 1 VVE nur der Kommission erteilt wird, muss diese auch allein für die Haushaltsausführung verantwortlich sein.

Art. III-407 UA I VVE ermächtigt nur „im Rahmen der zugewiesenen Mittel“ zur Ausführung des Haushaltsplans. Entsprechend dem Grundsatz der Spezialitäl dürfen die Mittel deshalb nur zu dem Zweck und nur in der Höhe ihrer Bewilligung verwendet werden. Zusätzlich zu dieser notwendigen Bedingung steht die Verwendung der veranschlagten Mittel regelmäßig unter der hinreichenden Bedingung, dass ein entsprechender sachlicher Rechtsakt erlassen ist (Art. I-53 Abs. 4 VVE). Darüber hinaus wird die Ausführung des Haushaltsplans durch die Haushaltsgrundsätze, insbesondere (aber nicht nur) durch den Grundsatz der Wirtschaftlichkeit begrenzt. Zwar werden die Mitgliedstaaten von Art. I-53 Abs. 6 VVE nicht als Adressaten des Wirtschaftlichkejtsgrundsatzes genannt, doch lässt sich sowohl aus Art. III-407 UA 1 VVE als auch aus dem Grundsatz der loyalen Zusammenarbeit nach Art. I-5 Abs. 2 VVE die Verpflichtung der Mitgliedstaten zu einer wirtschaftlichen Ausführung des Haushaltsplans ableiten.

In Ausnahme vom Grundsatz der Spezialität ermöglicht Art. III-407 UA 3 VVE sachliche Mittelübertragungen nach Maßgabe der Haushaltsordnung. Die derzeitigen Regelung der Art. 22 und 23 HO sind stark durch die Unterscheidung zwischen obligatorischen und nicht-obligatorischen Ausgaben geprägt. Mit der Aufhebung dieser Unterscheidung durch den VVE werden neue Regelungen in der Haushaltsordnung unabdingbar. Möglicherweise orientiert sich eine neue Haushaltsordnung wieder an der recht übersichtlichen und praktikablen Regelung der alten Haushaltsordnung. nach der Übertragungen von Artikel zu Artikel wegen der geringen politischen Bedeutung dieser Verschiebungen in die alleinige Verantwortung des jeweiligen Organs fielen und nur die Übertragungen von Kapitel zu Kapitel der Entscheidung der Haushaltsbehörde vorbehalten waren. ${ }^{39}$

39 Vgl. zu dieser Regelung Magiera, in: Grabitz/Hilf (Fn. 17). Art. 205 Rn. 9: Rossi (Fn. 23). S. $213 \mathrm{f}$. 


\section{Entlastung}

\section{a) Rechnungslegung}

Die von Art. III-407 UA 1 VVE hervorgehobene Eigenverantwortung der Kommission für die Ausführung des Haushaltsplans spiegelt sich auch in der von Art. III408 Abs. 1 VVE normierten Pflicht der Kommission wider, dem Parlament und dem Rat Rechenschaft für den Haushaltsvollzug zu geben. Zu diesem Zweck erstellt sie eine Haushaltsrechnung und eine Vermögensrechnung. Die Einzelheiten über den Umfang und das Verfahren der Rechnungslegung sind in der Haushaltsordnung festzulegen. ${ }^{40}$

\section{b) Evaluierungsbericht}

Neu ist die im VVE erstmals normierte P1licht der Kommission, dem Parlament und dem Rat einen Evaluierungsbericht vorzulegen. Bislang waren die Kommission und die anderen Organe nach Art. 276 Abs. 3 UA 2 EG nur aul gesondertes Ersuchen des Parlaments oder des Rates zu einem solchen Bericht verpflichtet. ${ }^{41}$

Der Evaluierungsbericht soll es dem Parlament und dem Ral im Entlastungsverfahren vereinfachen, die Ausführung des Haushalts durch die Kommission zu bewerten. $\mathrm{Zu}$ diesem Zweck soll die Kommission gesondert darlegen, ob und wie sie die Vorgaben des Parlaments oder des Rats umgesetzl hat, die ihr in vorausgegangenen Entlastungsverfahren auferlegt wurden. Der Begriff Vorgaben bezieht sich ausschließlich auf die von Art. III-409 Abs. 3 und Abs. 4 VVE angesprochenen Bemerkungen und Empfehlungen des Parlaments bzw. des Rates. Dass Art. III-408 UA 2 VVE nur pauschal auf Art. III-409 VVE und nicht exakt auf dessen Absäize 3 und 4 verweist, bedeutet nicht, dass der Evaluierungsbericht auch etwaige Vorgaben der laufenden Haushaltskontrolle zu berücksichtigen hat, denn zu solchen Vorgaben ist die Haushaltsbehörde nach dem VVE in Respekt vor der Eigenverantwortung der Kommission für die Haushaltsausführung nicht berechtigt.

\section{c) Entlastung und Entlastungsverfahren}

Das Verfahren, mit dem die Kommission für ihre Ausführung des Haushaltsplans durch das Parlament entlastet wird, wird von Art. III-409 VVE grob umschrieben und ist im Übrigen in der Haushaltsordnung insbesondere auch im Hinblick auf die Fristen, die von den beteiligten Organen im Interesse einer zügigen Entlastung zu beachten sind, zu konkretisieren. ${ }^{42}$

$40 \mathrm{Vgl}$. Art. $121 \mathrm{ff}$. HO 2002.

$41 \mathrm{Vgl}$. auch Art. 147 Abs. 2 HO 2002.

$42 \mathrm{Vgl}$. Art. I39 ff. HO 2002. 
Die Entlastung wird allein vom Parlament erteilt; der Rat ist seit 1977 nur noch empfehlend am Entlastungsverfahren beteiligt. ${ }^{43}$ Das Verfahren innerhalb des Parlaments wird in dessen neuer Geschäftsordnung auszugestalten sein. ${ }^{24}$ Es beschließt entsprechend Art. III-338 VVE mit absoluter Mehrheit. Dabei ist es (bei insofern offenem Wortlaut) nicht verpflichtet, die Entlastung zu erteilen, sondern kann diese auch verweigern. ${ }^{\text {t3 }}$ Ob es darüber hinaus auch teilweise oder bedingte Entlastungen aussprechen darf, ist rechtlich bislang nicht geklärt und wohl eher eine politisch zu beantwortende Frage, deren Bedeutung nicht überschätzt werden darf, weil das Parlament konkrete politische Kritik in seine Entlastungsbemerkungen einbringen und ihre Beachtung durch die Kommission nach Abs. 4 und Art. III-408 UA 2 VVE kontrollieren kann.

Die Entlastung erfolgt in der Form eines einfachen Parlamentsbeschlusses. Dem Beschluss wird eine Entschließung mit den Bemerkungen des Parlaments sowie der Beschluss über die Billigung der Haushaltsrechnung beigefügt. Sämtliche dieser Handlungen des Parlaments werden im Amtblatt veröffentlicht. ${ }^{\text {t6 }}$

Mit der Entlastung wird die externe Kontrolle der Haushaltsführung und damit zugleich der Haushaltskreislauf abgeschlossen. Ihr kommt eine doppelte Funktion zu: In rechnerischer Hinsicht bestätigt sie den Abschluss der Haushaltsrechnung des betreffenden Haushaltsjahres und schafft damit zugleich auch die Grundlage für die Rechnungsabschlüsse der Folgejahre, in politischer Hinsicht bewertet sie die Art und Weise der Haushaltsauführung der Kommission. ${ }^{47}$

Korrespondierend zur ausschließlichen Verantwortlichkeit der Kommission für die Haushaltsführung (Art. III-407 UA I S. I VVE) ist allein die Kommission Adressat der Entlastung. Sofern das Parlament auch anderen Organen Entlastung erteilt, ist diese keine Entlastung im Sinne des Art. III-407 VVE und kann deshalb auch nicht deren Rechtsfolgen erzeugen. ${ }^{48}$

\section{d) Rechtsfolgen der Entlastung}

In Bezug auf die Rechtsfolgen der Entlastung ist zwischen dem Entlastungsbeschluss und etwaigen in der Entschließung enthaltenden Bemerkungen des Parlaments zu differenzieren.

43 Zur Entwicklung des Entlastungsverfahrens s. Rossi (Fn. 23), S. 15I ff.

44 Vgl. Art. 93 i.V.m. Anlage V GO-EP, ABI. EG 1999 Nr. L 202/l.

45 Sir., wie hier schon Rossi (Fn. 23), S. 158 ff.: Kannengießer. DÖV 1995, S. 56; a.A. Bieber, in: Groeben/Schwarze (Fn. 13), An. 276 Rn. 12: Niedobilek, in: Streinz (Fn. I1), Ant. 276 Rn. 14: Schoo, in: Schwarze (Fn. 28), Art. 276 Rn. 11; Magiera, in: Grabitz/Hilf (Fn. 17). Art. 206 Rn. 7. Das Parlament hat bislang dreimal (für die Haushalısjahre 1982. 1985 und 1996) dic Entlastung verweigert.

46 Vgl. Niedobirek. in: Streinz (Fn. 1 1), An. 276 Rn. 12.

47 Vgl. Niedobitek, in: Streinz (Fn. 11), Art. 276 Rn. I m.w.N.

48 Ebenso Niedobritek, in: Streinz (Fn. 11), Art. 276 Rn. 7. 
Hinsichtlich des Entlastungsbeschlusses ist weiterhin zwischen seiner rechnerischen und seiner politischen Funktion zu unterscheiden. In Bezug auf seine rechnerische Funktion kommt dem Entlastungsbeschluss insofern Rechtswirkung zu, als er die Konten abschließt und die Vermutung für eine ordnungsgemiße Haushaltsführung der Kommission und der anderen beteiligten Organe begründet. Es dürfen keine weiteren Haushaltsoperationen für dieses Jahr gebucht oder Rechnungen geändert werden. ${ }^{49}$ In Bezug auf seine politische Funktion erzeugt der Entlastungsbeschluss dagegen keine Rechtsfolgen. Das gilt auch für den Fall seiner Verweigerung, die schon wegen abweichender Verfahrensregelung insbesondere nicht in ein Misstrauensvotum nach Art. III-340 VVE umgedeutet werden kann. Die politischen Folgen einer Entlastungsverweigerung können freilich weitreichend sein und - wie am 16.03.1999 - bis zum geschlossenen Rücktritt der Kommission führen.

Die in der Entschließung enthaltenen Bemerkungen des Parlaments sind dagegen mit eigenständigen Rechtsfolgen verbunden, die sich aus Abs. 3 sowie auch aus Art. 1Il-408 UA 2 VVE ergeben. Nach Abs. 3 muss die Kommission diesen Bemerkungen nachkommen, indem sie alle zweckdienlichen Maßnahmen ergreift. Art. 147 Abs. 1 HO erstreckt diese Rechtspflicht auch auf die anderen am Haushaltsvollzug beteiligten Organe. Diese Maßnahmen müssen nach Art. III-408 UA 2 VVE in nächsten Evaluierungsbericht geschildert werden. Bei seinen an die Kommission gerichteten Bemerkungen differenziert das Parlament nach Schwere des festgestellten Mangels und unterscheidet dementsprechend zwischen stets zu befolgenden Aufforderingen, grundsätzlich zu befolgenden Empfehlumgen und nur zu prüfenden Vorschlägen. ${ }^{50}$

\section{e) Ergänzende Berichtspflichten}

Art. Ill-409 Abs. 4 VVE normiert Berichtspflichten der Kommission, die ein entsprechendes Ersuchen des Parlaments oder des Rates voraussetzen. Die Bedeutung dieser Berichtspflichten wird durch die grundsätzliche Verpflichtung der Kommission, zur Umsetzung der Vorgaben des Parlaments und des Rates stets im Evaluierungsbericht nach Art. III-408 UA 2 VVE Stellung zu nehmen, abnehmen, aber nicht verloren gehen, denn sie ermöglicht dem Parlament und dem Rat nach wie vor gezielte Nachfragen. Außerdem müssen die Berichte nach Abs. 4 auch dem Rechnungshof zugeleitet werden, während die Evaluierungsberichte - vorbehaltlich einer entsprechenden Regelung in der Haushaltsordnung - nur dem Parlament und dem Rat vorgelegt werden müssen.

49 H.M., vgl. Bieber, in: Groeben/Schwarze (Fn. 13), Ar. 276 Rn. 17; differenzierend Kannengießer, DÖV 1995, S. 58; Rossi (Fn. 23), S. 163.

50 Einzelheiten bei Theato/Graf. Das Europäische Parlament und der Haushalt der Europäischen Gemeinschaften, 1994, S. 130 f. 
f) Begleitende Haushaltskontrolle

Art. III-409 Abs. 2 VVE schlieBlich begründet ein umfassendes Auskunftsrecht des Parlaments gegenüber der Kommission, das inhaltlich und zeitlich über das eigentliche Entlastungsverfahren hinausreicht und damit den verfassungsrechtlichen Grundstein für eine begleitende Haushaltskontrolle legt. Es ist allerdings seinem Wortlaut nach auf die Ausgabentätigkeit beschränkt. die freilich regelmäßig im Mittelpunkt des Kontrollinteresses des Parlaments steht. Das Auskunftsrecht kann durch die Haushaltsordnung oder etwa durch eine Vereinbarung zwischen dem Parlament und der Kommission ausgestaltet werden, ohne dass damit eine weitere Berufung des Parlaments auf Abs. 2 ausgeschlossen wäre. Art. 131 HO sieht u.a. Vierteljahresberichte vor, die im sog. ..Notenboom-Verfahren" im Haushaltskontrollausschuss des Parlaments geprüft werden. ${ }^{51}$ Darüber hinaus sind die Modalitäten der Informationsübermittlung in einer am 01.01.2001 in Kraft getretenen Rahmenvereinbarung über die Beziehungen zwischen dem Parlament und der Kommission geregelt. ${ }^{52}$

\section{Trilog}

Ein weiteres Novum im primären Recht der Union enthält Art. III-414 VVE. Die Bestimmung institutionalisiert und konstitutionalisiert das als Trilog bezeichnete Treffen der benannten Organpräsidenten und konkretisiert zugleich den Grundsatz der Organtreue ${ }^{53}$ für das Haushalısverfahren. ${ }^{54}$

Der Trilog findet nur im gegenseitigen Einvernehmen der drei Organpräsidenten statl, wobei der Kommission eine koordinierende Initiativaufgabe, nicht aber eine bindende Initiativbefugnis übertragen wird. ${ }^{55}$

Der Trilog kann ,im Rahmen der nach diesem Kapitel vorgesehenen Haushaltsverfahren" durchgeführt werden, also nicht nur im Rahmen des Haushaltsverfahrens im engeren Sinne nach Art. III-404 VVE, sondern auch im Rahmen der Aufstellung des mehrjährigen Finanzrahmens nach Art. I-55 VVE und Art. 1Il-402 VVE, ${ }^{56}$ der Überschreitung der monatlichen Zwölftel im Nothaushaltsverfahren nach Art. III405 Abs. 2 VVE, des Entlastungsverfahrens nach Art. III-409 VVE und auch im Rahmen des Erlasses der Haushaltsordnung und anderer Finanzvorschriften nach Art. III-412 VVE.

51 Vgl. Theato/Graf (Fn. 50). S. 135 f.; Rossi (Fn. 23), S. 149.

52 ABI. EG 2001 Nr. C 121/122.

53 Vgl. Art. I-19 Abs. 2 S. 2.

54 Der Trilog geht auf die Gemeinsame Erklärung des Parlaments, des Rates und der Kommission vom 30.06.1982 zuruick, mil dem der bereits beim EuGH anhängig gemachte Streit um den Haushaltsplan von 1982 beigclegt werden konnte, und hat ihr Vorbild in entsprechenden Regelungen der Interinstitutionellen Vereinharungen von 1988, 1993 und 1999 (S. Anhang III zur Interinstitutionellen Vereinbarung vom 6.05.1999. ABI. $1999 \mathrm{Nr}$. C 172/1: und jüngst Anhang 11 zur Interinstitutionellen Vereinbarung vom 14.06.2006. ABl. 2006 Nr. C 139/1). Vgl. Rossi (Fn. 23). S. 34 f.

$55 \mathrm{Vgl}$. aher Interinstitutionelle Vercinbanung 2006. Anm. F 26 Abs. 5 (ABI. 2006 Nr. C 139/4).

56 Vgl. auch Art. III-402 Abs. 5. 
Seine Aufgabe besteht darin, den gesamten Haushaltskreislauf („die Durchführung dieses Kapitels ${ }^{\text {}}$ ) zu erleichtern. Zu diesem Zweck sollen die Präsidenten kompromisssuchend und -fördernd auf ihre Organe einwirken. Als Mittel sind ihnen dabei ,alle erforderlichen Maßnahmen" an die Hand gegeben, die sich freilich nur auf die Förderung der Abstimmung und Annäherung der Standpunkte der Organe und nicht etwa auf deren Erfolg beziehen. Hervorzuheben ist dennoch, dass diese Maßnahmen unter dem Vorbehalt der verfassungsrechllichen Befugnisse der Organe und ihrer Teile stehen und insbesondere auch die Ausgestaltung der organinternen Entscheidungsfindung zu respektieren haben. Im Ergebnis sollen die Präsidenten ihre Organe dazu anhalten, ihre jeweiligen Verfahrensrechte im Haushaltskreislauf entsprechend dem Grundsat $z$ der Organtreue mit Rücksicht auf die Rechte der jeweils anderen Organe und vor allem mit dem Ziel auszuüben, den verfassungsrechtlich vorgesehenen Haushaltskreislauf zu ermöglichen. Insofern artikuliert die Bestimmung mittelbar ein Koordinierungs- und Kooperationsgebot für die drei am Haushaltsverfahren beteiligten Organe, ${ }^{57}$ das allerdings letztlich nicht justitiabel ist und insofern nur als soft law zu qualifizieren ist. ${ }^{58}$

\section{Weitere Vorschriften}

\section{Europäischer Rechnungshof}

In Bezug auf den Europäischen Rechnungshof bringt der VVE keine bedeutenden Veränderungen. Art. I-31 VVE konstituiert die Organqualität des Rechnungshofs und umreißt die Grundsäıze seiner Aufgaben. Zugleich bestimmt er die Zusammensetzung des Rechnungshofs und garantiert seinen Mitgliedern die unabhängige Aufgabenausübung. ${ }^{59}$ Hinsichtlich der Aufgaben des Rechnungshofs wird die Norm durch Art. III-384 und III-385 VVE konkretisiert. Ihre Bedeutung erschöpft sich insoweit darin, Modifizierungen bestimmter Kerngehalte dem ordentlichen Änderungsverfahren nach Art. IV-443 VVE vorzubehalten. Konkrete Befugnisse und Pflichten ergeben sich nur aus Art. III-384 und III-385 VVE. Konsequenz der von Art. I-31 Abs. 3 S. 1 VVE angeordneten Zusammensetzung des Rechnungshofs ist, dass die Zahl seiner Mitglieder mit jeder Erweiterung anzupassen ist. Einzelheiten der Ernennung regelt Art. III-385 VVE.

Die in Art. I-31 Abs. 3 S. 2 VVE normierte Unabhängigkeitsgarantie, die ihrerseits die Grundlage für die Autorität des Rechnungshofs ist, entfaltet Wirkungen für die Union und die Mitgliedstaaten auf der einen Seite und für die Mitglieder des Rechnungshofs auf der anderen Seite: Erstere dürfen die Tätigkeit des Rechnungshofs nicht durch Weisungen oder sonstige Maßnahmen beeinflussen, letzlere dürfen Weisungen nicht entgegennehmen oder gar anfordern und müssen auch sonst jede

57 Fischer (Fn. 9), S. 492.

58 Fischer (Fn. ), S. 492.

59 Zum Europäischen Rechnungshof vgl. umfassend Freytag. Der Europäische Rechnungshof, Institution, Funktion und politische Wirkung, 2004, passim. 
Handlung unterlassen, die mit ihren Aufgaben unvereinbar ist (vgl. Art. III-385 Abs. 3 VVE). Sie sind allein auf das allgemeine Wohl der Union verpflichtet.

\section{Betrugsbekämpfung}

Die Bestimmung des Art. III-415 VVE über die Betrugshekämpfung unterscheidet sich von Art. 280 EG vor allem in zwei Punkten: Zum einen erstreckt Abs. 1 S. 2 die effektive Schutzwirkung der zu ergreifenden Maßnahmen nun auch auf die Einrichtungen der Union, zum anderen ist der Vorbehalt für das Strafrecht und die Strafrechtspflege in den Mitgliedstaaten in Abs. 4 entfallen. Damit steht der Union nun eine Ermäichtigungsgrundlage auf dem Gebiet des Strafrechts und des Strafverfahrensrechts zur Verfügung.

\section{Ausblick}

Aus legistischer Sicht erscheint die Finanz- und Haushaltsverfassung des VVE durchaus gelungen. Die Vorschriften lassen sowohl durch ihre systematische Anordnung als auch durch ihre Inhalte die rechtlichen Rahmenbedingungen für die Aufstellung des Haushaltsplans, seine Ausführung und deren Enllastung klar erkennen. Ungeachtet des Schicksals der oder einer Europäischen Verfassung bleibt insoweit zu hoffen, dass das geltende Gemeinschaftsrecht rasch unter Berücksichtigung der finanz- und haushaltsrechllichen Bestimmungen des VVE novelliert werden. Dann allerdings müssen sich diese Vorschriften in der Praxis erst noch bewähren. Die am Haushaltsverfahren beteiligten Organe werden ihre neuen rechtlichen Befugnisse und politischen Einflussmüglichkeiten erst im Laufe der Zeit in einer Weise zu nutzen wissen, die nicht nur ihren jeweils eigenen Interessen dient, sondern auch den reibungslosen Ablauf des Haushaltsverfahrens insgesamt gewährleistet. Das gilt vor allem auch für das Verfahren im Vermittlungsausschuss, in dem es in besonderer Weise auf das Verhandlungsgeschick der in ihn entsendeten Vertreter des Rats und des Parlaments ankommen wird. Insofern gilt für die Verfassungsordnung der Europäischen Union dasselbe wie für die Verfassungsordnung eines jeden ihres Mitgliedstaats: Sie stellt einen verbindlichen und dadurch verlässlichen Rahmen auf, der die unterschiedlichen politischen Akteure zugleich legitimiert und limitiert. ${ }^{60}$

60 Zur funktionalen Betrachtung der Europäischen Verfassungsordnung vgl. Mïller-Graff, EWS 2002, S. 206, . S. $209 \mathrm{ff}$. 